\title{
ASSESSING SLEEP USING HIP AND WRIST ACTIGRAPHY
}

\section{Authors}

James A Slater, GradDipSleepSc ${ }^{1}$; Thalia Botsis, BSc(Hons) ${ }^{1}$; Jennifer Walsh, $\mathrm{PhD}^{1}$; Stuart King, $\mathrm{BSc}^{1}$; Leon M Straker, $\mathrm{PhD}^{2}$; Peter R Eastwood, $\mathrm{PhD}^{1}$

\author{
Affiliations \\ ${ }^{1}$ Centre for Sleep Science, School of Anatomy, Physiology and Human Biology, University \\ of Western Australia, Perth, Australia \\ ${ }^{2}$ School of Physiotherapy and Exercise Science, Curtin University, Perth, Australia
}

\section{Category}

Original article report of research

\section{Correspondence to}

Winthrop Professor Peter Eastwood

Centre for Sleep Science School of Anatomy, Physiology \& Human Biology

M309, University of Western Australia

35 Stirling Highway Crawley 6009, Western Australia, Australia

Telephone: +61 893461706

Fax: +61 893462034

Email: peter.eastwood@health.wa.gov.au

\section{Word count}




\section{SUMMARY OF NEW FINDINGS}

- Compared to polysomnography (PSG), a GTX3+ Actigraph placed on the wrist provides a moderately valid measure of sleep as defined by its high sensitivity (90\%), although it has poor wake detection as defined by its low specificity (46\%).

- Compared to PSG, a GTX3+ Actigraph placed on the hip does not provide a valid or reliable measure of sleep as defined by its low specificity (14\%).

- Measures of total sleep time and sleep efficiency were similar on wrist compared to PSG, while measures of sleep onset latency and wake after sleep onset were significantly different on wrist compared to PSG. Measures of total sleep time, sleep efficiency, sleep onset latency and wake after sleep onset were significantly different on hip actigraphy compared to PSG. 


\section{ABSTRACT AND KEYWORDS}

Wrist actigraphy is commonly used to measure sleep, and hip actigraphy is commonly used to measure activity. It is unclear whether hip-based actigraphy can be used to measure sleep. This study assessed the validity of wrist actigraphy and hip actigraphy compared to polysomnography (PSG) for the measurement of sleep.

108 healthy young adults (22.7 \pm 0.2 years) wore hip and wrist GTX3+ Actigraph during overnight PSG. Measurements of total sleep time (TST), sleep efficiency (SE), sleep onset latency (SOL) and wake after sleep onset (WASO) were derived and compared between wrist actigraphy, hip actigraphy and PSG. Sensitivity, specificity and accuracy of wrist actigraphy and hip actigraphy for each variable were derived from epoch-by-epoch comparison to PSG. Compared to PSG: TST and SE were similar by wrist actigraphy but overestimated by hip actigraphy (both by 14\%); SOL was underestimated by wrist actigraphy and hip actigraphy (by 39 and 80\%, respectively); WASO was overestimated by wrist actigraphy and underestimated by hip actigraphy (by 34 and 65\%, respectively). Compared to PSG the sensitivity, specificity and accuracy of wrist actigraphy were 90, 46 and 84\%, respectively; and of hip actigraphy were 99, 14 and 86\%, respectively.

This study showed that using existing algorithms, a GTX3+ Actigraph worn on the hip does not provide valid or accurate measures of sleep, mainly due to poor wake detection. Relative to the hip, a wrist worn GTX3+ Actigraph provided more valid measures of sleep, but with only moderate capability to detect periods of wake during the sleep period.

Keywords: actigraphy, polysomnography, sleep, activity, validation, accuracy 


\section{INTRODUCTION}

Wrist-based actigraphy has been widely used to measure sleep. ${ }^{1}$ The operational premise is that periods of sleep are accompanied by minimal movement, and periods of wakefulness are accompanied by relatively more movement, and that such movement can be detected by an accelerometry based activity monitor. ${ }^{2}$ The non-dominant wrist has been the most commonly used site of monitor placement, however other locations have been utilized including the shoulder, ${ }^{3}$ leg, ${ }^{4}$ and trunk/hip. ${ }^{5,6,7}$ Most validation studies comparing actigraphy to the gold standard measure of polysomnography (PSG) have used non-dominant wrist placement. $^{8}$ Hip-based actigraphy is commonly used to measure physical activity. ${ }^{1}$ The hip placement is usually favored as it records the large amplitudes associated with vertical trunk movements of interest to estimating energy expenditure during activities such as walking, ${ }^{9}$ although it performs poorly at detecting arm movement and load carrying activities. ${ }^{10}$ Large scale population health studies such as the Canadian Health Measures Survey (CHMS) and the US National Health and Nutritional Examination Survey (NHANES) have used hip actigraphy to provide valuable information on patterns of physical activity. ${ }^{9,10}$ Many physical activity studies have recorded data continuously over several days. If hip actigraphy were found to be a valid measure of sleep, it would enable the use of one device worn at the hip to measure 24hour activity and sleep. This could facilitate population scale investigations of both physical activity and sleep health. Further, it could lower barriers to use and improve the cost effectiveness of actigraphy for physical activity and sleep.,11

To date, very few studies have compared hip actigraphy to wrist actigraphy for the measurement of sleep. ${ }^{6,7,12}$ A recent study assessed the GTX3+ placed at the hip, with SOMNOWatch plus ${ }^{\circledR}$ device at the hip and wrist, and compared these to PSG on overall 
sleep measures but did not evaluate the data epoch-by-epoch, and used PSG sleep scoring rules which have been superseded. ${ }^{7}$

Our study aimed to evaluate the validity of wrist actigraphy and hip actigraphy for measuring sleep in healthy young adults, by measuring sleep using GTX3+ Actigraphs placed on the wrist and hip with simultaneous laboratory based PSG, and comparing overall sleep measures as well as epoch-by-epoch data.

\section{MATERIALS AND METHODS}

\section{Subjects}

A total of 108 volunteers were recruited for this study, 57 males and 51 females. Participants had a mean age of $22.7 \pm 0.2$ years and their BMI was $25.5 \pm 5.6 \mathrm{~kg} \cdot \mathrm{m}^{-2}$, and ranged from 18.3 to $46.7 \mathrm{~kg} \cdot \mathrm{m}^{-2}$. All participants were healthy young adults participating in the 23-year follow up of the Western Australian Pregnancy Cohort (Raine) Study, an ongoing longitudinal study that is for the first time assessing sleep using PSG. ${ }^{13}$ Ethics approval for the study was granted by The University of Western Australia Human Research Ethics Committee (RA/4/1/5202). All participants gave voluntary informed consent.

\section{Protocol}

Participants attended the Centre for Sleep Science at University of Western Australia where they were had simultaneous overnight recordings of PSG, wrist actigraphy and hip actigraphy. Studies took place on weeknights. Participants arrived early in the evening to complete ongoing cohort physical and cognitive assessments. Later in the evening participants were set up for PSG and actigraphy as close to their bedtime as practicable and 
encouraged to engage in their usual home-based settling behaviors. Participants woke of their own accord, typically between 0530 and 0700 .

\section{Polysomnography}

Comprehensive in-laboratory PSG was performed using the Compumedics Grael system (Compumedics, Abbotsford, Victoria, Australia). The recording montage was in accordance with the American Academy of Sleep Medicine (AASM) recommended technical specifications and included electroencephalogram (F3-M2, F4-M1, C3-M2, C4-M1, O1-M2, O2-M1 positioned according to the $10 / 20$ system), ${ }^{14}$ left and right electrooculogram (EOG), mental-submental electromyogram (EMG), bilateral tibial EMG, nasal pressure transducer airflow (Salter Labs, California, USA), thermistor-based oronasal airflow (Compumedics, Abbotsford, Victoria, Australia), chest and abdominal respiratory inductance plethysmography (Compumedics, Abbotsford, Victoria, Australia), arterial $\mathrm{SpO}_{2}$ (Compumedics, Abbotsford, Victoria, Australia), body position (Compumedics, Abbotsford, Victoria, Australia), decibel meter and infrared video (VIVOTEK, New Taipei City, Taiwan).

Data were analysed using Compumedics ProFusion PSG3 software. Scoring of studies was initiated at "lights off” and ended at "lights on”. These times were automatically noted by a light detector, marked on the PSG recording and confirmed on analysis. Data were scored according to American Academy of Sleep medicine 2012 rules for the scoring of sleep. ${ }^{14}$ This included use of 30 second epochs for sleep staging, assigning epochs a state of sleep or wake, documenting respiratory and limb movement events and generating indices of the frequency of these events. A single trained sleep scientist scored all PSG recordings.

\section{Actigraphy}


Actigraphs were time synchronized to the PSG systems prior to use. Actigraphy recordings were performed using GT3X+ activity monitors (Actigraph, Florida, USA), a small (4.6 cm x $3.3 \mathrm{~cm}$ x $1.5 \mathrm{~cm}$ ), lightweight (19 grams) triaxial solid state accelerometer based activity monitor. Data were digitized by a 12-bit analog to digital converter at $30 \mathrm{Hertz}(\mathrm{Hz})$ and with a $0.25 \mathrm{~Hz}$ high pass filter and a $2.5 \mathrm{~Hz}$ low pass filter.

The wrist Actigraph was worn on the non-dominant wrist. The hip Actigraph was held in place with an elastic belt so that it was positioned on participant's right side in line with the right thigh.

Wrist actigraphy and hip actigraphy data were downloaded and analysed using the ActiLife software (Actigraph 2012, ActiLife 6.8). The time of "lights off” and "lights on” were taken from the PSG recording. Actigraphy data were scored in one-minute epochs as awake or asleep according to Sadeh's algorithm ${ }^{15}$ using data from the vertical axis. A single trained scientist scored all actigraphy recordings.

\section{Data analysis}

The primary sleep variables collected were: total sleep time (TST: minutes of sleep between "lights off” and "lights on”); sleep efficiency (SE: minutes of total sleep time divided by minutes available for sleep between "lights off" and "lights on", then multiplied by 100 to obtain a percentage); sleep onset latency (SOL: number of minutes from "lights out" to the first epoch scored as sleep) and wake after sleep onset (WASO: number of minutes awake between first epoch scored as sleep and “lights on”). 
Measurements of TST, SE, SOL and WASO derived from the wrist Actigraph, hip Actigraph and PSG were compared using repeated measures ANOVA on ranks as the data were not normally distributed. A post-hoc Tukey Test was applied. The Bland Altman method was used to assess the magnitude (bias) and pattern (proportional or systematic) of differences in sleep measures between each actigraphy device and PSG ${ }^{16,17}$ and associated descriptive statistics and confidence intervals were used to assess measurement differences between devices. Intraclass correlation coefficients on a two way single measures mixed model were calculated to compare sleep parameters between the two devices and PSG.

The sensitivity, specificity and accuracy of the wrist and hip Actigraph was determined for each participant by comparing the state of sleep or wake scored by the Actigraph to the state of sleep or wake scored by the gold standard measure of PSG. Sensitivity was defined as the proportion of epochs correctly scored by actigraphy as sleep. Specificity was defined as the proportion of epochs correctly scored as wake. Accuracy was defined as the proportion of all epochs correctly scored as wake or sleep. Paired t-tests were used to assess the agreement of epoch-by-epoch comparisons. Where data were not normally distributed non-parametric tests were utilized.

As PSG requires 30 second epochs ${ }^{14}$ and actigraphy required one minute epochs ${ }^{15}$ it was necessary to transform the standard 30-second scored PSG epochs into one-minute epochs of either wake or sleep: where both 30 second PSG epochs had been scored as sleep, the transformed one minute epoch was classified as sleep; where one or more of the 30 second PSG epochs had been scored as wake, the transformed one minute epoch was classified as wake. 
All statistical analyses were undertaken using IBM SPSS version 22 (IBM, New York, USA) and SigmaStat version 3.5 (SysStat Software Inc., San Jose, CA, USA).

\section{RESULTS}

\section{Overall sleep measures}

Compared to PSG (Table 1): TST and SE were similar when measured by wrist actigraphy but were overestimated by hip actigraphy by $14 \%$ and $14 \%$ respectively $(P<0.05)$; SOL was underestimated by both hip and wrist actigraphy by $39 \%$ and $80 \%$, respectively $(P<0.05)$; and WASO was overestimated by wrist by 34\% $(P<0.05)$ and underestimated by hip actigraphy by $65 \%(P<0.05)$.

Intraclass correlations (Table 2) indicated moderate relationships between: PSG and both wrist and hip actigraphy for TST and SE; and PSG and wrist actigraphy for WASO. Poor relationships were noted for: PSG and hip actigraphy for TST and WASO; and for both wrist actigraphy and hip actigraphy for SOL.

\section{Bland Altman plots of measurement differences}

Bland Altman plots of each of the 4 sleep variables are shown in Figures 1-4 and the associated descriptive statistics are shown in Table 3. TST was similar when measured by wrist actigraphy compared to PSG (bias $0.3 \pm 74.3$ minutes, $P=0.97$ ). In contrast, hip actigraphy significantly overestimated TST by $54.3 \pm 59.7$ minutes $(P<0.05)$. The slope of the relationship indicated a proportional bias such that the degree of overestimation became less as TST increased $\left(r^{2}=0.03, P<0.05\right)$. 
SE was similar when measured by wrist actigraphy compared to PSG $(-1.4 \pm 10.5 \%, P=$ 0.19). In contrast, hip actigraphy significantly overestimated SE by $11.5 \pm 10.5 \%(P<0.05)$. The slope of the relationship indicated a proportional bias such that the degree of overestimation decreased as SE increased $\left(r^{2}=0.56, P<0.05\right)$.

SOL was underestimated by both wrist and hip actigraphy, by $-7.3 \pm 18.0$ and $-15.1 \pm 19.4$ minutes, respectively $(P<0.05)$. The slopes of both relationships indicated a proportional bias such that the degree of underestimation increased as SOL increased (wrist $\mathrm{r}^{2}=0.11, P<$ 0.05; hip $\left.r^{2}=0.34, P<0.05\right)$.

WASO was systematically overestimated by wrist actigraphy by $16.1 \pm 32.4$ minutes $(P<$ 0.05). In contrast, WASO was underestimated by hip actigraphy by $-31.0 \pm 36.6$ minutes $(P<$ 0.05). The slope of the relationship indicated a proportional bias such that the degree of underestimation increased as WASO increased $\left(r^{2}=0.38, P<0.05\right)$.

\section{Epoch-by-epoch comparison to PSG}

Sensitivity, specificity and accuracy of both devices relative to PSG are shown in Table 4. While overall accuracy of both devices was good, and sensitivity high for both devices, the specificity was only moderate for wrist actigraphy and poor for hip actigraphy.

\section{DISCUSSION}

This is the first study to simultaneously measure sleep using wrist actigraphy, hip actigraphy and PSG. The major findings were: (i) that wrist actigraphy correctly scored sleep with high sensitivity, moderate specificity and overall high accuracy; and (ii) that hip actigraphy correctly scored sleep with high sensitivity, unacceptably low specificity and an overall high 
accuracy. In other words, both wrist actigraphy and hip actigraphy were good at detecting periods of sleep but moderate to poor at detecting periods of wakefulness during sleep.

For any measure to be regarded as accurate it should have high sensitivity and specificity. ${ }^{18}$ Though guidelines and methods vary, recommendations for specificity start at a generous minimum of at least $40 \%$ and ideally greater than $60 \%$ should be achieved. ${ }^{19,20}$ The moderate specificity of wrist actigraphy and poor specificity of hip actigraphy reflect an inability of these devices to correctly detect, or agree with, EEG-defined periods of wakefulness. Actigraphy is a surrogate measure of sleep that is reliant on the presence of movement to indicate wakefulness and lack of movement to indicate sleep. Thus, periods of wakefulness not accompanied by movement will incorrectly be scored as sleep by actigraphy. ${ }^{21,22,23}$ The data from the present study suggests that this misclassification is exacerbated when measuring movement with an Actigraph positioned at the hip.

Previous studies of simultaneous hip and wrist actigraphy to assess sleep have reported varied results. Hjorth et al. compared a GT3X+ placed on the non-dominant wrist to the same type of device placed on the hip in children and found the mean sensitivity, specificity and accuracy of hip relative to wrist actigraphy to be $99.8,46.9$ and $87.6 \%$ respectively. ${ }^{12}$ Similar to the findings in the present study, Hjorth et al. reported that hip actigraphy overestimated TST and SE compared to wrist actigraphy. ${ }^{12}$ In another study, Paavonen et al. found that hip actigraphy agreed with wrist actigraphy for $75 \%$ of the time spent awake and $95 \%$ of the time spent asleep. ${ }^{5}$ While not directly comparing actigraphy to PSG, the Paavonen et al. study highlights the decreased capacity of hip actigraphy, relative to wrist actigraphy, to detect wakefulness. ${ }^{5}$ Enomoto et al. trialed an algorithm developed specifically for a hip device which was compared to PSG; and reported a sensitivity of $89.4 \%$ and specificity of 
$58.2 \% .{ }^{6}$ The reasons for the increased specificity in this relative to the present study are not clear, but could relate to (i) the use of an actigraphy device that utilized 2 minute epochs, such that periods of wake less than one minute could have been scored as sleep, thus increasing the amount of sleep scored or (ii) scoring of PSG studies using Rechtschaffen and Kales rules which might result in less WASO scored by PSG than would be the case using the contemporary AASM scoring rules ${ }^{24,25}$ which were used in our study.

Zinkan et al. assessed wrist actigraphy and hip actigraphy in a sample of participants aged between 18 and 75 years, using SOMNOWatch plus ${ }^{\circledR}$ devices on the wrist and hip, as well as GTX3+ placed on the hip only, with all actigraphy devices scored using the Cole-Kripke algorithm and recorded simultaneously to PSG. ${ }^{7}$ They did not perform an epoch-by-epoch comparison to obtain sensitivity, specificity and accuracy. This is a different method and sample to our sample of healthy young adults aged close to 23 years, using a GTX3+ at both the wrist and hip, actigraphy scored using the Sadeh algorithm, recorded simultaneously to PSG both overall and epoch-by-epoch. Zinkan et al. concluded that wrist actigraphy was superior to hip actigraphy for measuring sleep, ${ }^{7}$ which agrees with our findings.

In our study wrist actigraphy and hip actigraphy both underestimated SOL (by 38 and 80\%, respectively). Such an underestimation of SOL is consistent with previous studies. ${ }^{5,8,26}$ Actigraphy generally underestimates SOL because individuals may be awake, yet inactive, for a period of time prior to EEG defined sleep. The absence of motion will result in actigraphic scoring algorithms determining “sleep”, leading to underestimation of SOL, as observed in this study. Hip actigraphy underestimated SOL significantly more than wrist actigraphy, most likely as a result of wrist movements occurring while the hip remained still. The Bland-Altman plots of SOL for wrist actigraphy vs. PSG and hip actigraphy vs. PSG 
revealed proportional biases: as the average SOL increased, the difference between the measurement of SOL by wrist and hip actigraphy and PSG increased. Thus, the higher the average SOL, the more likely it is that the Actigraph (wrist or hip) will underestimate SOL relative to PSG. In other words, the ability of the Actigraph (whether worn on the wrist or the hip) to accurately measure SOL is reduced with increasing wakefulness. This may be partly explained by differences in physiological variables measured. ${ }^{7,19}$

Hip actigraphy performed poorly in determining periods of wakefulness during the night. Hip actigraphy over-scored sleep (as evident by the overestimation of TST and SE), and under scored wake (as evident by the underestimation of WASO). The very low specificity of hip actigraphy also affected the hip actigraphy-derived measurement of WASO and SE. Relative to PSG, hip actigraphy underestimated WASO by 65\% on average and overestimated SE by $13 \%$ on average. Further, the Bland-Altman plots comparing hip actigraphy and PSG for WASO and SE in the present study both revealed a proportional bias: as WASO and SE increased, the difference between the measurement of these variables by hip actigraphy and PSG increased. Thus, hip actigraphy underestimated WASO and SE by a greater amount as the magnitude of these variables increases. These are most likely a consequence of the inability of hip actigraphy to correctly identify periods of wakefulness. Consistent with the findings from this study, Hjorth et al. also reported an overestimation of SE (when compared to wrist actigraphy), which was likely due to the low specificity observed in their study. ${ }^{12}$

Unlike hip actigraphy, wrist actigraphy overestimated WASO by 32\% on average, measuring 15 minutes more WASO compared to PSG. This, in combination with the underestimation of TST, led to wrist actigraphy underestimating SE by $1 \%$ on average. These results are consistent with those from a recent study published by Meltzer et al. which reported a 
significant overestimation of WASO and underestimation of TST and SE by wrist actigraphy relative to PSG. ${ }^{27}$ As previously discussed, physiological variables measured to determine sleep are different for actigraphy and PSG. Wrist movements can occur during EEG-defined sleep. As such the movements are not necessarily indicative of wake, and so may be mistakenly scored as "wake” by the scoring algorithm. This would account for some of the increased WASO measured by wrist actigraphy.

The methods required to analyse and compare actigraphy to the gold standard PSG measure of sleep may cause or exacerbate differences. Transformation of PSG epochs from 30 seconds to one minute, and alignment of these with actigraphy, is one possible source of difference. Actigraphy scoring algorithms weight movements according to activity in nearby epochs. ${ }^{19}$ Movements could occur within a single epoch or across multiple epochs, so the timing of a movement could impact not only the epoch it occurs in, but also nearby epochs, and the way in which these epochs are transformed by the algorithm. Further, PSG is an intrusive process, and performing PSG may not provide a realistic sampling of participant's sleep. While a strength of our study is that actigraphy was compared to the gold standard sleep measure of PSG, a limitation is that one night of PSG does not allow for assessment of the reliability of actigraphy, or its capability for detecting sleep-wake patterns over multiple nights. $^{23}$

Actigraphy algorithms were created and validated for recording of movement on one axis of movement once per second, ${ }^{15}$ and studies using the algorithms have continued to use them this way. Technology has evolved since the original validation studies. The devices used in our study are capable of recording 30 times per second on three axes. Algorithm advancements to utilize the higher resolution and multiple axes recorded could improve 
accuracy ${ }^{8,18}$ by incorporating more subtle movements or movements that are better detected in different axes of movement. ${ }^{4}$ Such improvements could be especially useful for hip-based actigraphy. $^{15}$

The Sadeh algorithm used in this study was developed for a device worn on the wrist. The direction, duration and intensity of movements at the wrist are different to movements at the hip. Our study has shown that use of the Sadeh algorithm with a GTX3+ Actigraph at the hip is neither a valid or accurate measure of sleep, due to inability to recognize wake-related movements during sleep. It however possible that adjusting the algorithm or device for differences in movement could increase the specificity of hip actigraphy. ${ }^{6}$

Despite the participants in our study being healthy young adults, 10 participants had an apnoea-hypopnoea index greater than 5 , and 3 participants had a periodic leg movement index greater than 10. Excluding these participants from the cohort did not alter the sensitivity, specificity and accuracy of wrist actigraphy or hip actigraphy. AASM guidelines on the clinical use of wrist actigraphy recommend the technique to be suitable for measuring sleep time and documenting sleep patterns in healthy adults and children. ${ }^{8}$ Our findings on healthy young adults support these guidelines. 


\section{ACKNOWLEDGEMENTS AND DISCLOSURE}

We acknowledge and thank Raine Study participants and Raine Study Team for cohort management and data collection. The authors have indicated no conflict of interest in this research. The authors are gracious for the ongoing support for this project.

This research was not funded by industry. The Raine Study 23 year follow up is supported by NHMRC project grants 10277449, 1021858, 1031617 and 1044840. Core funding for cohort management was provided by the University of Western Australia, the Telethon Institute for Child Health Research, Raine Medical Research Foundation, University of Western Australia Faculty of Medicine, Dentistry and Health, Women’s and Infant's Research Foundation and Curtin University.

Professors Straker and Eastwood were funded by National Health and Medical Research Council of Australia (NHMRC) Senior Research Fellowships (1019980, 1042341). 


\section{FIGURE LEGENDS}

\section{Figure 1 - Bland Altman plots of Total Sleep Time}

Plots of total sleep time displaying wrist actigraphy compared to PSG on the left and hip actigraphy compared to PSG on the right, $n=108$. Difference = actigraphy - PSG. Average $=$ $($ actigraphy + PSG $) / 2$. Solid line $=$ mean of difference Dashed lines $= \pm 2$ SD. Perfect agreement is indicated by a mean difference of zero.
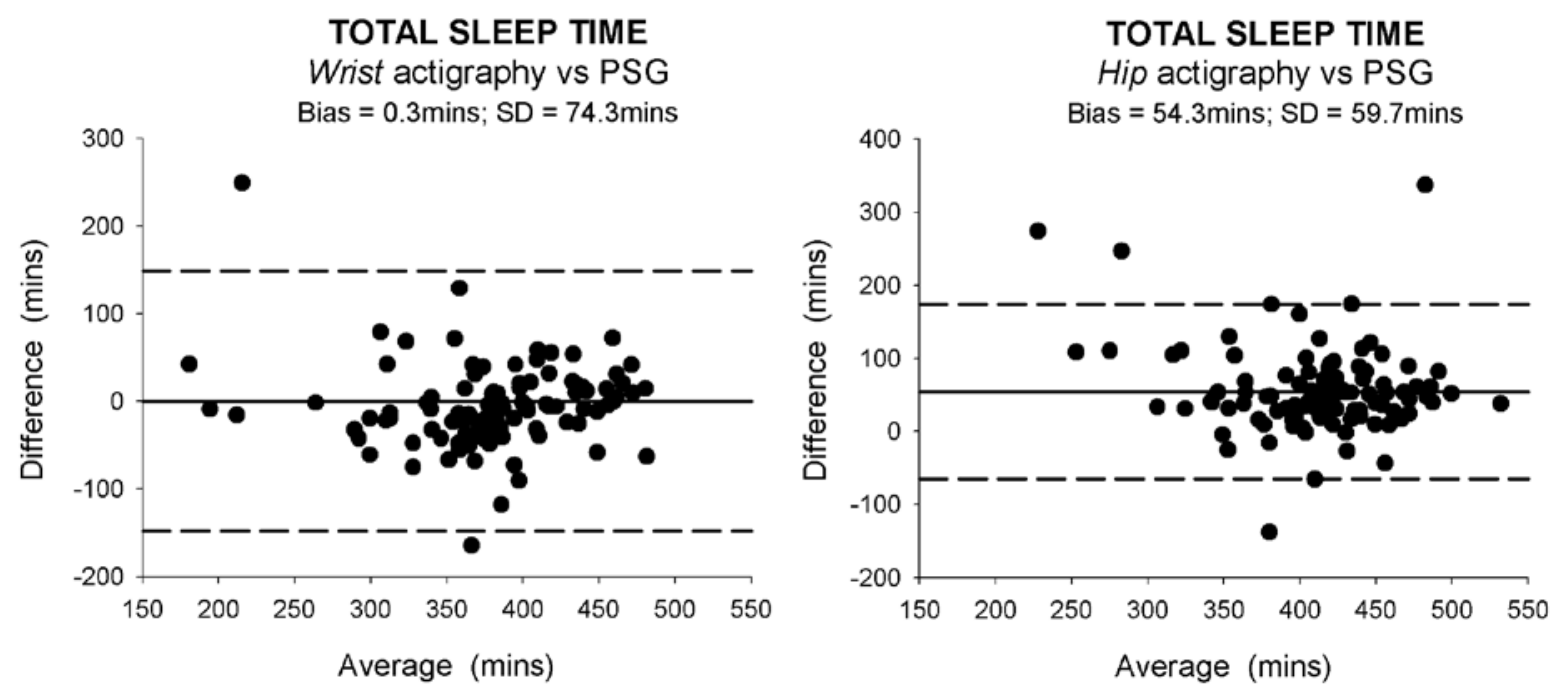


\section{Figure 2 - Bland Altman plots of Sleep Efficiency}

Plots of sleep efficiency displaying wrist actigraphy compared to PSG on the left and hip actigraphy compared to PSG on the right, $n=108$. Difference = actigraphy - PSG. Average $=$ $($ actigraphy + PSG $) / 2$. Solid line $=$ mean of difference Dashed lines $= \pm 2 S D$. Perfect agreement is indicated by a mean difference of zero.
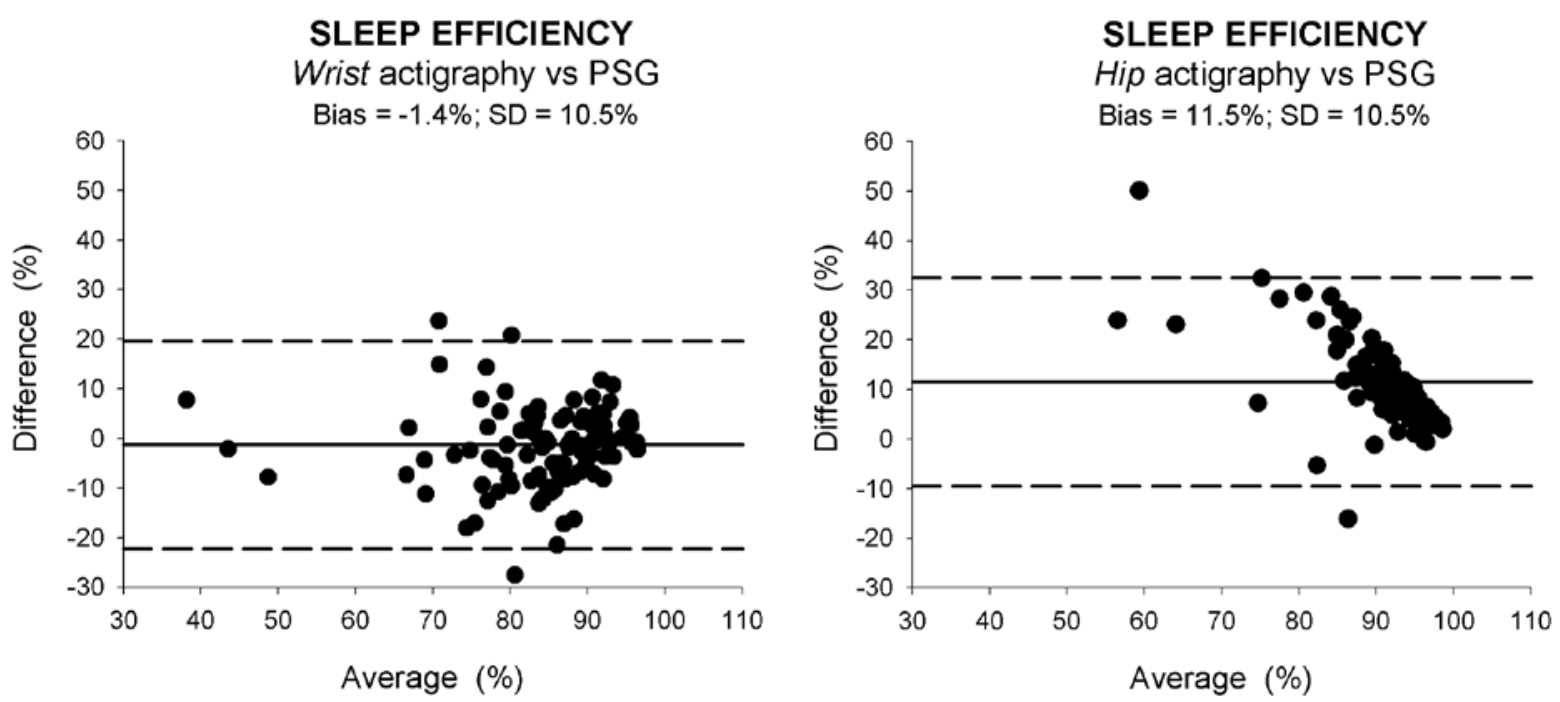


\section{Figure 3 - Bland Altman plots of Sleep Onset Latency}

Plots of sleep onset latency displaying wrist actigraphy compared to PSG on the left and hip actigraphy compared to PSG on the right, $n=108$. Difference = actigraphy - PSG. Average $=$ $($ actigraphy + PSG $) / 2$. Solid line $=$ mean of difference Dashed lines $= \pm 2 S D$. Perfect agreement is indicated by a mean difference of zero.
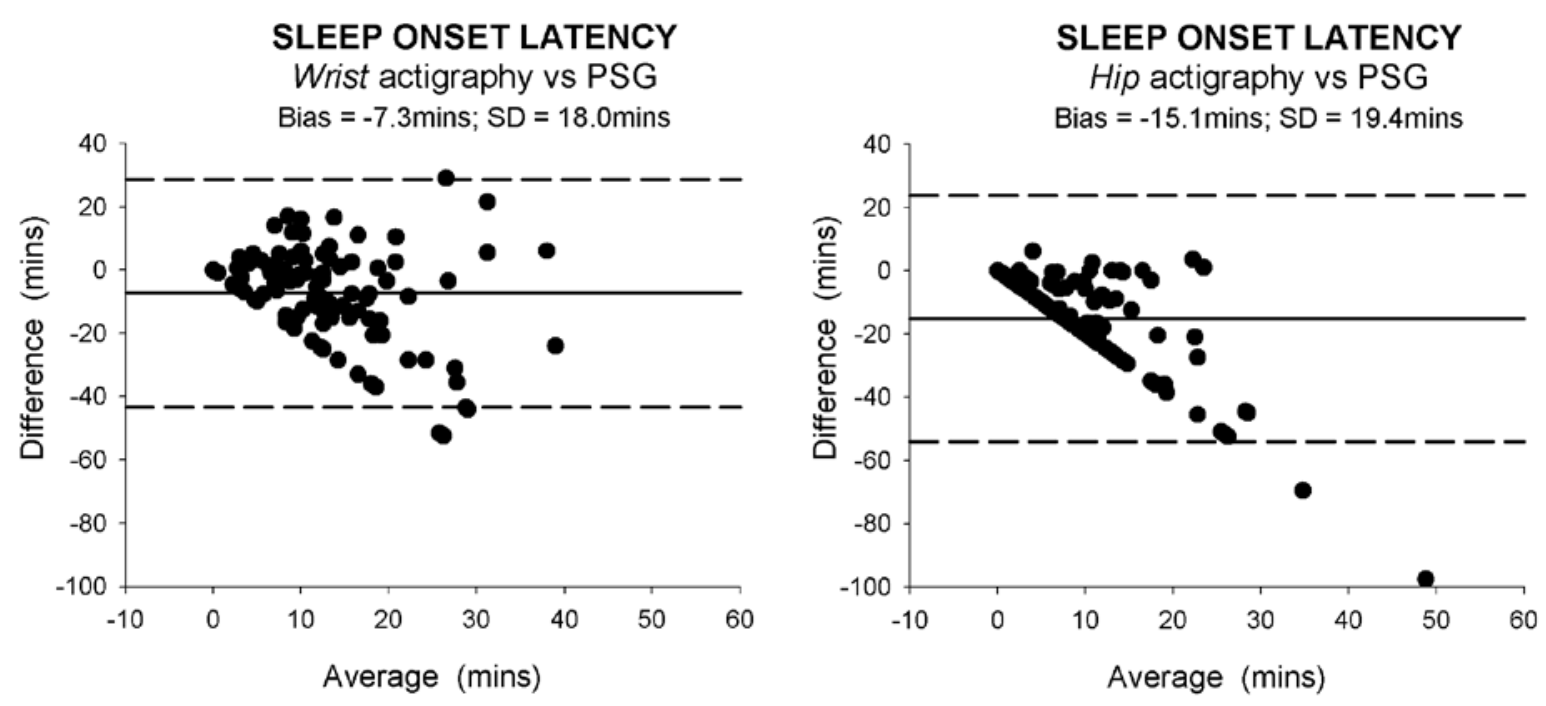


\section{Figure 4 - Bland Altman plots of Wake After Sleep Onset}

Plots of wake after sleep onset displaying wrist actigraphy compared to PSG on the left and hip actigraphy compared to PSG on the right, $n=108$. Difference = actigraphy - PSG.

Average $=($ actigraphy + PSG $) / 2$. Solid line $=$ mean of difference Dashed lines $= \pm 2$ SD .

Perfect agreement is indicated by a mean difference of zero.
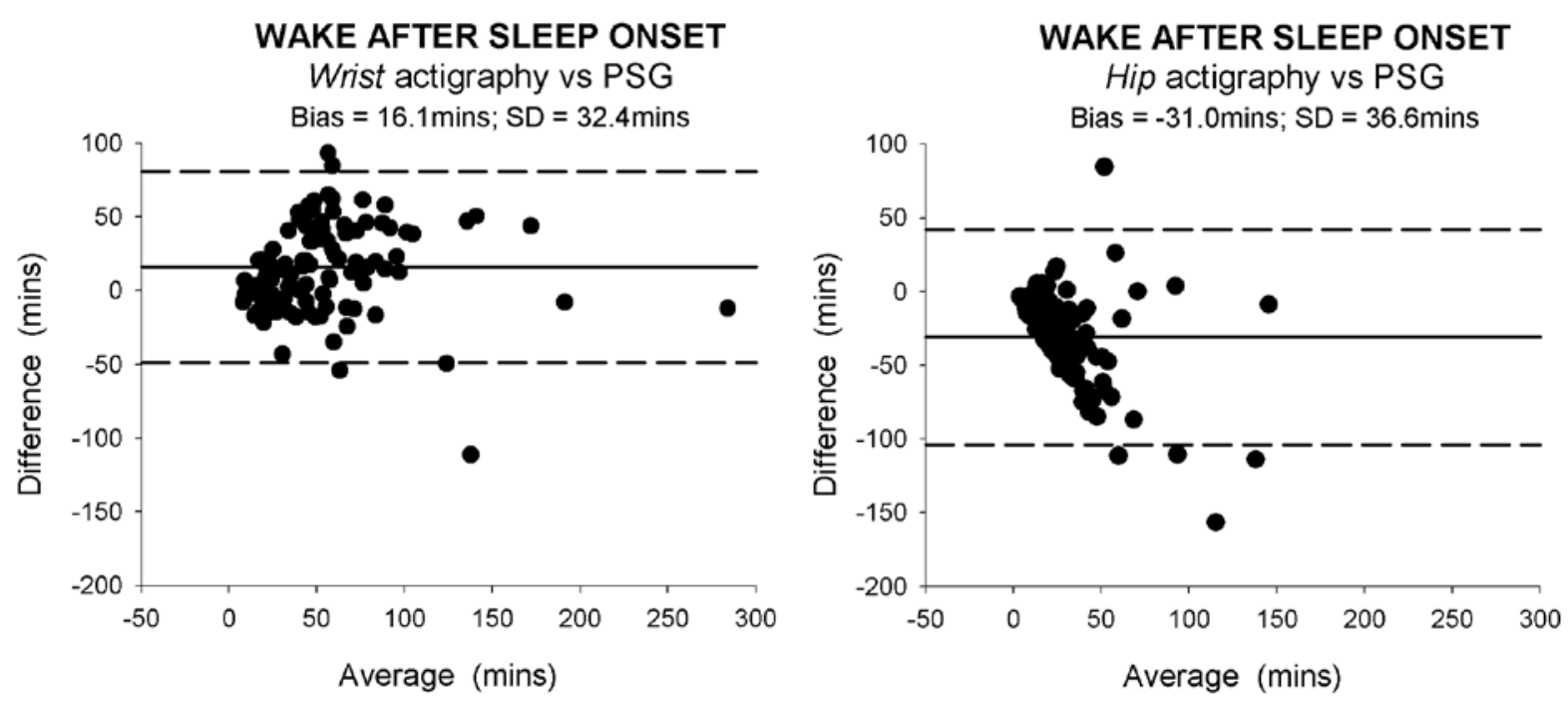


\section{TABLES}

Table 1 - Summary sleep measures

\begin{tabular}{lccc}
\hline Measure & PSG & Wrist & Hip \\
\hline TST (minutes) & $382.4 \pm 63.8$ & $382.7 \pm 85.0$ & $436.7 \pm 54.4 * \dagger$ \\
SE (\%) & $84.6 \pm 12.2$ & $83.3 \pm 10.8$ & $96.1 \pm 5.2 * \dagger$ \\
SOL (minutes) & $18.8 \pm 18.0$ & $11.5 \pm 13.1 * \dagger$ & $3.7 \pm 9.3 * \dagger$ \\
WASO (minutes) & $47.8 \pm 42.4$ & $63.9 \pm 44.9 * \dagger$ & $16.8 \pm 22.5 * \dagger$ \\
\hline
\end{tabular}

*vs PSG $(P<005)$, post hoc Tukey test; †vs wrist actigraphy $(P<0.05)$, post hoc Tukey test. $\mathrm{PSG}=$ polysomnography; $\mathrm{TST}=$ total sleep time; $\mathrm{SE}=$ sleep efficiency; $\mathrm{SOL}=$ sleep onset latency; WASO = wake after sleep onset. 
Table 2 - Intraclass correlations between actigraphy and PSG

\begin{tabular}{lcc}
\hline Measure & Wrist actigraphy & Hip actigraphy \\
\hline TST & 0.51 & 0.35 \\
SE & 0.59 & 0.22 \\
SOL & 0.32 & 0.05 \\
WASO & 0.68 & 0.30 \\
\hline
\end{tabular}

PSG = polysomnography; TST = total sleep time; $\mathrm{SE}=$ sleep efficiency; $\mathrm{SOL}$ = sleep onset latency; WASO = wake after sleep onset. 
Table 3 - Bland Altman statistics for sleep measure differences between actigraphy and PSG

\begin{tabular}{|c|c|c|c|c|c|c|c|c|}
\hline & \multicolumn{4}{|c|}{ Wrist actigraphy } & \multicolumn{4}{|c|}{ Hip actigraphy } \\
\hline & TST & SE & SOL & WASO & TST & SE & SOL & WASO \\
\hline & (mins) & (\%) & (mins) & (mins) & (mins) & (\%) & (mins) & (mins) \\
\hline Mean difference & 0.3 & -1.4 & -7.3 & 16.1 & 54.3 & 11.5 & -15.1 & -31.0 \\
\hline Standard deviation & 74.3 & 10.5 & 18.0 & 32.4 & 59.7 & 10.5 & 19.4 & 36.6 \\
\hline Upper limit & 14.3 & 0.7 & 3.9 & 22.2 & 65.6 & 13.5 & -11.5 & -24.1 \\
\hline Lower limit & -13.7 & -3.3 & -10.7 & 9.9 & 43.0 & 9.5 & -18.8 & -37.6 \\
\hline
\end{tabular}

PSG = polysomnography; $\mathrm{TST}=$ total sleep time; $\mathrm{SE}=$ sleep efficiency; $\mathrm{SOL}=$ sleep onset latency; WASO = wake after sleep onset. Shaded variables represent mean differences that were significantly different to PSG. 
Table 4 - Sensitivity, specificity, and accuracy

Wrist compared to PSG Hip compared to PSG

\begin{tabular}{lcc}
\hline Sensitivity (\%) & $89.7 \pm 8.2$ & $98.6 \pm 2.3 *$ \\
Specificity (\%) & $45.6 \pm 20.3$ & $14.3 \pm 14.7 \dagger$ \\
Accuracy (\%) & $84.1 \pm 8.1$ & $86.2 \pm 8.5 *$ \\
\hline
\end{tabular}

*vs wrist compared to PSG $(P<0.05)$, Wilcoxon signed rank test. †vs wrist compared to PSG $(P<0.05)$, paired t-test. PSG = polysomnography; sensitivity = \% of PSG sleep epochs correctly identified as sleep by actigraphy; specificity = \% of PSG wake epochs correctly identified as wake by actigraphy; accuracy = \% of PSG sleep and wake epochs. 


\section{REFERENCES}

1. Mathie MJ, Coster AC, Lovell NH, Celler BG. Accelerometry: providing an integrated, practical method for long-term, ambulatory monitoring of human movement. Physiol Meas. 2004;25(2):R1-20.

2. Tryon WW. Nocturnal activity and sleep assessment. Clinical Psychology Review. 1996;16(3):197-213.

3. Adkins KW, Goldman SE, Fawkes D, Surdyka K, Wang L, Song Y, et al. A pilot study of shoulder placement for actigraphy in children. Behav Sleep Med. 2012;10(2):138-47.

4. Middelkoop HA, van Dam EM, Smilde-van den Doel DA, Van Dijk G. 45-hour continuous quintuple-site actimetry: relations between trunk and limb movements and effects of circadian sleep-wake rhythmicity. Psychophysiology. 1997;34(2):199-203.

5. Paavonen EJ, Fjälberg M, Steenari M-R, Aronen ET. Actigraphy placement and sleep estimation in children. Sleep. 2002;25(2):235-7.

6. Enomoto M, Endo T, Suenaga K, Miura N, Nakano Y, Kohtoh S, et al. Newly developed waist actigraphy and its sleep/wake scoring algorithm. Sleep and Biological Rhythms. 2009;7:17-22.

7. Zinkan M, Berger K, Hense S, Nagel M, Obst A, Koch B, et al. Agreement of different methods for assessing sleep characteristics: a comparison of two actigraphs, wrist and hip placement, and self-report with polysomnography. Sleep Med. 2014 May 28. doi: 10.1016/j.sleep.2014.04.015 [Epub ahead of print].

8. Morgenthaler TI, Lee-Chiong T, Alessi C, Friedman L, Aurora RN, Boehlecke B, et al. Practice parameters for the clinical evaluation and treatment of circadian rhythm sleep disorders. An American Academy of Sleep Medicine report. Sleep. 2007;30(11):144559. 
9. Troiano RP, Berrigan D, Dodd KW, Masse LC, Tilert T, McDowell M. Physical activity in the United States measured by accelerometer. Medicine and science in sports and exercise. 2008;40(1):181-8.

10. Colley RC, Garriguet D, Janssen I, Craig CL, Clarke J, Tremblay MS. Physical activity of Canadian adults: accelerometer results from the 2007 to 2009 Canadian Health Measures Survey. Health reports. 2011;22(1):7-14.

11. Kinder JR, Lee KA, Thompson H, Hicks K, Topp K, Madsen KA. Validation of a hipworn accelerometer in measuring sleep time in children. Journal of pediatric nursing. 2012;27(2):127-33.

12. Hjorth MF, Chaput JP, Damsgaard CT, Dalskov SM, Michaelsen KF, Tetens I, et al. Measure of sleep and physical activity by a single accelerometer: Can a waist-worn Actigraph adequately measure sleep in children? Sleep and Biological Rhythms. 2012;10:328-35.

13. McKnight CM, Newnham JP, Stanley FJ, Mountain JA, Laundau LI, Beilin LJ, et al. Birth of a cohort-the first 20 years of the Raine study. Med J Aust. 2012;197(11):60810.

14. Berry RB, Brooks R, Gamaldo CE, Harding SM, Marcus CL, Vaughn BV, et al. The AASM Manual for the Scoring of Sleep and Associated Events. Darien, IL: American Academy of Sleep Medicine; 2012.

15. Sadeh A, Sharkey KM, Carskadon MA. Activity-based sleep-wake identification: an empirical test of methodological issues. Sleep. 1994;17(3):201-7.

16. Bland JM, Altman DG. Statistical methods for assessing agreement between two methods of clinical measurement. Lancet. 1986;1(8476):307-10.

17. Bland JM, Altman DG. Comparing methods of measurement: why plotting difference against standard method is misleading. Lancet. 1995;346(8982):1085-7. 
18. Ancoli-Israel S, Cole R, Alessi C, Chambers M, Moorcroft W, Pollak CP. The role of actigraphy in the study of sleep and circadian rhythms. Sleep. 2003;26(3):342-92.

19. Tryon WW. Issues of validity in actigraphic sleep assessment. Sleep. 2004;27(1):15865.

20. Sadeh A. The role and validity of actigraphy in sleep medicine: an update. Sleep Med Rev. 2011;15(4):259-67.

21. Blood ML, Sack RL, Percy DC, Pen JC. A comparison of sleep detection by wrist actigraphy, behavioral response, and polysomnography. Sleep. 1997;20(6):388-95.

22. Marino M, Li Y, Rueschman MN, Winkelman JW, Ellenbogen JM, Solet JM, et al. Measuring sleep: accuracy, sensitivity, and specificity of wrist actigraphy compared to polysomnography. Sleep. 2013;36(11):1747-55.

23. Sadeh A, Acebo C. Sleep Medicine Rev. 2002;6(2):113-24.

24. Moser D, Anderer P, Gruber G, Parapatics S, Loretz E, Boeck M, et al. Sleep classification according to AASM and Rechtschaffen \& Kales: effects on sleep scoring parameters. Sleep. 2009;32(2):139-49.

25. Grigg-Damberger MM. The AASM Scoring Manual four years later. J Clin Sleep Med. 2012;8(3):323-32.

26. de Souza L, Benedito-Silva AA, Pires ML, Poyares D, Tufik S, Calil HM. Further validation of actigraphy for sleep studies. Sleep. 2003;26(1):81-5.

27. Meltzer LJ, Walsh CM, Traylor J, Westin AML. Direct comparison of two new actigraphs and polysomnography in children and adolescents. Sleep. 2012;35(1):15966. 